\title{
Improving compliance with iron infusion therapy in the treatment of chronic anemia in haemodialysis patients with chronic kidney disease
}

Amith Nuti

United Kingdom

\begin{abstract}
This quality improvement project was conducted at the haemodialysis unit in the paediatric nephrology department at Noah's Ark Children's Hospital, Cardiff. Stakeholders involved were the medical and nursing staff at the haemodialysis unit, responsible for the care of children with chronic kidney disease CKD.
\end{abstract}

Anaemia is prevalent among children with CKD. Iron infusion is administered to such children with chronic anaemia. Children on haemodialysis attending the Children's Kidney Center receive iron infusion if they satisfy the criteria based on haemoglobin and serum ferritin values according to departmental guidelines. This involves measurement of c-reactive protein and serum ferritin prior to iron administration. High iron exposure is detrimental to end organ function and hence warrants regular monitoring in conjunction with CRP, another inflammatory marker. We suspect that some children may be receiving iron infusions despite being iron replete. Also, we may be over-investigating these children with anaemia.

We identified all children receiving iron infusion in the haemodialysis unit over a four week period. We retrospectively enquired blood investigations done, prior to and after iron infusion. Blood investigations lagged on pre and post infusion times.

We devised a checklist for nursing staff to follow, primarily looking at set times for measuring haemoglobin, serum ferritin, and CRP during the month (at the start of the first and third week of the month) and also tabulating the ferritin values that would trigger frequency of iron infusions. These were aimed to:

1. Prevent iron overloading in patients with chronic anemia

2. Regularise the checking of bloods in those receiving iron infusions

3. Empower the nursing staff to independently take decisions on iron infusion delivery.

The strategy for change encompassed multiple PDSA cycles.

Plan: empower decision making on iron infusion by haemodialysis nursing staff

Do: formulate a checklist for iron infusion based on the recommended set values of ferritin, CRP and haemoglobin

Study: analyse adherence to checklist in three months time

Act: make appropriate changes to workplace behaviour based on findings of the PDSA cycle

We analysed 13 patient episodes prior to the intervention and a total of 19 patient episodes after the improvement cycles. The checklist was improved based on feedback obtained after the first PDSA cycle. A second cycle showed that investigations done were optimised. The third cycle showed improved adherence and compliance with prevention of over-treatment with iron infusion. There was $100 \%$ adherence to the investigations done prior to infusion and complied well with the department guidelines. This meant that the required number of blood tests were done on a more regular basis and it did not exceed from those done previously.

Nursing behaviour with regard to initiation and maintenance of iron infusion became more independent. This empowered nursing decision making skills and consequently freed doctor-time. It also resulted in improving team morale and ultimately patient safety by mitigating human errors.

For any QI project, interventions should be carefully designed. Stakeholder buy-in and easy accessibility of the intervention improves sustainability. Multiple PDSA cycles and incorporating stakeholder feedback into the cycle are key to success. 


\section{Problem}

Incidence and prevalence of end stage renal disease in children worldwide is 5 to 15 and 15 to 300 per million population respectively. Anemia is prevalent among children with CKD and its prevalence increases from $1 \%$ of stage 3 chronic kidney disease to $33 \%$ of stage 5 chronic kidney disease. Iron infusion is administered to such children with chronic anemia.

\section{Background}

Children on hemodialysis attending the Children's Kidney Centre receive an iron infusion if they satisfy the criteria based on hemoglobin and serum ferritin values determined by department guidelines. This involves the measurement of CRP and serum ferritin prior to iron administration. High iron exposure is detrimental to end organ function and hence regular monitoring of serum ferritin. However, serum ferritin is also an acute phase reactant and hence has to be paired with CRP to avoid iron injection during inflammatory process.

We have a protocol on iron infusion for patients with chronic anemia secondary to chronic kidney disease on hemodialysis. However, we suspect that some children may be receiving iron infusions despite being iron replete. Also, we may be over-investigating these children with anemia.

See supplementary file: ds3431.pdf - "Iron infusion protocol"

\section{Baseline measurement}

We identified all patients receiving iron infusion in the hemodialysis unit over a four week period (March 2014 - April 2014). We retrospectively enquired blood investigations done prior to and after iron infusion. Blood investigations were based as per the departmental protocol.

There were four patients (on hemodialysis) identified who received the iron infusion at different times when they attended the Children's Kidney Centre for their dialysis sessions. The blood investigations that had been done immediately prior and post infusion were logged. Blood investigations lagged on pre and post infusion times.

We devised a checklist for nursing staff to follow that primarily looked at set times for measuring hemoglobin, serum ferritin, and CRP during the month (at the start of the first and third week of the month), as well as tabulating the ferritin values that would trigger frequency of iron infusions.

See supplementary file: ds3662.doc - "Check-list for iron infusion in children with chronic kidney disease on hemodialysis"

\section{Design}

We identified particular time periods during the month (targeting the start of first and third week of the month) to check on serum ferritin and CRP on patients who have received and completed a course of iron infusion for anemia. By adhering to the checklist prompts, we hope to:

1. Prevent iron overloading in patients with chronic anemia

2. Regularise the checking of bloods in those receiving iron infusion

3. Empower the nursing staff to independently take decisions on iron infusion for chronic anemia in patients with chronic renal failure.

\section{Strategy}

Plan: Prevent iron overloading. Empower decision making by nursing staff in the haemodialysis unit regarding iron infusions for chronic anaemia.

Do: Setting up of iron infusion checklist to be followed for every patient who warrants iron infusion for chronic anaemia with recommendations based on set values of serum ferritin, CRP, and haemoglobin.

Study: Analyse the blood investigations done on patients after the checklist has been followed in the work place after few months. Examine patient episodes.

Act: Make appropriate changes to work place behaviour based on feedback from PDSA cycle. Iron infusions given during set times of the week, and investigations were done on fortnightly basis.

See supplementary file: ds4464.JPG - "PDSA4"

\section{Post-measurement}

Data was collected again after three months of implementation of the checklist. Total number of iron infusions given and the blood investigations done during the period July 2014 - September 2014 were collated.

Changes were made to the checklist to incorporate the timing of blood investigations and iron infusions whilst on maintenance iron infusions.

A total of 13 patient episodes were examined in the first PDSA cycle. In the third cycle, 19 patient episodes were examined.

See supplementary file: ds $4443 . d o c$ - "iron infusion compliance checklist"

\section{Lessons and limitations}

Blood investigations done for patients receiving iron infusion were more regularised and were not excessive in comparison to the beginning of the project. 


\section{BMJ Quality Improvement Reports}

Nursing staff behaviour has become more independent as regards to initiation and maintenance of iron infusion for chronic anemia. This frees up valuable doctor time, while at the same time empowers nursing decision making. This also helps in improving team morale and ultimately patient safety by mitigating human factors.

Feedback from one of the PDSA cycles meant that one patient could have benefited from receiving less iron infusion as the iron stores were adequate.

The improvement cycle was repeated after two months which showed improved compliance and adherence to checklist. One hundred percent of patient episodes examined were correctly investigated and given infusions as per guideline after the third cycle.

The checklist was applied to limited number of patient episodes. We plan to roll it out to all patients in the department.

\section{Conclusion}

Patients with chronic kidney disease on hemodialysis regularly need iron infusions based on their iron stores and degree of anemia. This is regulated by regular checking of hemoglobin, serum ferritin, and CRP blood values. We aimed to empower hemodialysis nursing staff to manage the iron infusions based on a checklist of ferritin and hemoglobin values thereby making them more independent. This had the added benefit of freeing doctor time.

The checklist formatting was improved after the first PDSA cycle and the second cycle showed that the number of investigations done pre and post infusions were optimised. It also revealed that one of the patient episode did not need further maintenance iron infusion at that point in time as the blood stores for iron were optimal.

We completed another PDSA cycle with the necessary interventions. The third cycle showed improved compliance and adherence with prevention of over treatment with iron infusion.

Performing multiple PDSA cycles, reflecting on feedback and making necessary changes to the service are key to any quality improvement project.

\section{References}

1. Van Bokhoven MA, Kok G, van der Weijden T. Designing a quality improvement intervention: a systematic approach. Quality Saf Health Care 2003; 12:215-20

2. Evaluating the quality of medical care. Donabedian A; Milbank Mem Fund Q 1966; 44(3): supp:166-206.

3. Departmental clinical guidelines on iron infusion for chronic anemia in children on haemodialysis for chronic kidney diasease, University hospital of Wales, Cardiff and vale university health board.

\section{Declaration of interests}

None

\section{Acknowledgements}

Staff at haemodialysis unit - S/N Matthew Pernas, S/N Helen Carlin, S/N Lauren Holland, S/N Margaret Lewis, Dr Rajesh Krishnan 